\title{
Medição em Organizações de Software: Observações do Estado da Prática
}

\author{
Gleison Santos \\ Programa de Pós-Graduação em Informática - Universidade Federal do Estado do Rio \\ de Janeiro (UNIRIO) - Av. Pasteur, 458, Urca, CEP 22290-240 - Rio de Janeiro, RJ \\ gleison.santos@uniriotec.br
}

Resumo. $O$ processo Medição deve ser implementado por empresas que objetivam o Nível $F$ do Modelo de Referência do MPS.BR (MR-MPS). As organizações tem liberdade de decidir a melhor forma de implementar tal processo desde que seja aderente ao MR-MPS. Este artigo apresenta um relato sobre a implementação do processo Medição do MR-MPS em organizações a partir de observações do estado da prática em organizações que implementaram este processo. Espera-se, dessa forma, contribuir positivamente para a implementação deste processo em iniciativas de melhoria de processo de software baseadas no MR-MPS.

\begin{abstract}
Organizations aiming to achieve the MPS.BR level F must implement the Measurement process present in the MPS Reference Model (MR-MPS). Organizations may implement this process according to their needs provided that it is adherent to the MR-MPS expected results. This paper presents observations about the state of practice of Measurement Process implementation collected on organizations that have implemented the MRMPS. We expect these observations to positively influence software process improvement initiatives based on the MR-MPS.
\end{abstract}

\section{Introdução}

O MPS.BR é programa para Melhoria de Processo do Software Brasileiro [SOFTEX 2009a]. Um de seus principais componentes é o Modelo de Referência (MR-MPS), que compreende 7 diferentes níveis de maturidade como uma combinação entre processos e sua capacidade: A (Em Otimização), B (Gerenciado Quantitativamente), C (Definido), D (Largamente Definido), E (Parcialmente Definido), F (Gerenciado) e G (Parcialmente Gerenciado) - sendo o nível $G$ o mais inicial e o nível A o mais avançado. A cada novo nível de maturidade mais processos devem ser implementados pelas organizações, assim como novos atributos de processo (que determinam o nível de capacidade).

$\mathrm{O}$ processo Medição, presente no Nível $\mathrm{F}$ do MR-MPS, pressupõe que as organizações definam medidas a partir de seus objetivos organizacionais e as utilizem para tomada de decisão. Este processo tem um papel importante em implementações de melhoria de processo de software por estar por trás de aspectos importantes na evolução aos níveis de maturidade subsequentes que culminam nos níveis de alta maturidade (A e B). Mesmo atendendo aos requisitos previstos pelo modelo, uma organização pode não atingir todo o potencial possível. Por isso, implementações deficientes deste processo podem ter impacto indesejado no futuro dos programas de melhoria das organizações.

Este artigo apresenta um relato a partir de observações do estado da prática em organizações que implementaram o processo Medição. Não é objetivo deste artigo, no 
entanto, ser um substituto do Guia de Implementação do MR-MPS [SOFTEX 2009b], mas complementá-lo em alguns aspectos. A partir da descrição de problemas comumente enfrentados, espera-se contribuir positivamente para a implementação deste processo em iniciativas de melhoria de processos de software baseadas no MR-MPS.

O conjunto de observações e melhores práticas relacionadas à implementação de Medição apresentadas neste artigo foram coletadas a partir de situações vivenciadas em organizações de software que implementaram ou foram avaliadas no MPS. O autor deste artigo foi avaliador, adjunto ou líder, em 20 avaliações MPS.BR em organizações que solicitaram avaliação de nível igual ou superior ao F. Além disso, é implementador do MPS desde 2004 com experiência de consultoria em várias empresas e participou ativamente da equipe da Área de Qualidade da COPPE/UFRJ avaliada no nível E do MRMPS [SANTOS, 2009a; 2009b].

Além desta introdução, este artigo está organizado em 5 seções. A Seção 2 apresenta os principais resultados esperados do MR-MPS relacionados a medição até o nível C. A Seção 3 descreve o conjunto de observações e melhores práticas relacionadas à definição de um plano de medição enquanto o foco da Seção 4 é a execução do conteúdo deste plano. A Seção 5 apresenta observações sobre a calibração da base de estimativas de uma organização. Por fim, as considerações finais são apresentadas na Seção 6.

\section{Medição no MR-MPS}

O processo Medição, no nível F do MR-MPS, tem como propósito “coletar, armazenar, analisar e relatar os dados relativos aos produtos desenvolvidos e aos processos implementados na organização e em seus projetos, de forma a apoiar os objetivos organizacionais" [SOFTEX 2009a]. A Tabela 1 apresenta os seus resultados esperados.

Tabela 1 - Resultados Esperados do Processo Medição [SOFTEX 2009a]

\begin{tabular}{|c|l|}
\hline Sigla & \multicolumn{1}{c|}{ Texto dos Resultados Esperados } \\
\hline MED 1 & $\begin{array}{l}\text { Objetivos de medição são estabelecidos e mantidos a partir dos objetivos de negócio da } \\
\text { organização e das necessidades de informação de processos técnicos e gerenciais. }\end{array}$ \\
\hline MED 2 & $\begin{array}{l}\text { Um conjunto adequado de medidas, orientado pelos objetivos de medição, é identificado e } \\
\text { definido, priorizado, documentado, revisado e, quando pertinente, atualizado. }\end{array}$ \\
\hline MED 3 & Os procedimentos para a coleta e o armazenamento de medidas são especificados. \\
\hline MED 4 & Os procedimentos para a análise das medidas são especificados. \\
\hline MED 5 & Os dados requeridos são coletados e analisados. \\
\hline MED 6 & Os dados e os resultados das análises são armazenados. \\
\hline MED 7 & $\begin{array}{l}\text { Os dados e os resultados das análises são comunicados aos interessados e são utilizados para } \\
\text { apoiar decisões. }\end{array}$ \\
\hline
\end{tabular}

Medição é um processo de apoio e, dessa forma, pode e deve ser utilizado em conjunto com outros processos. Em especial, há um relacionamento deste processo com a gerência de projetos e também como a institucionalização do programa de melhoria de processos de uma organização. A Tabela 2 apresenta alguns destes resultados esperados presentes nos processos Gerência de Projetos (GPR), Definição do Processo Organizacional (DFP) e Avaliação e Melhoria do Processo Organizacional (AMP).

Os níveis de maturidade do MR-MPS têm uma natureza evolutiva, representada, em parte, pelos Atributos de Processo (AP), que determinam a capacidade do processo. Espera-se que num primeiro momento (nível G), os processos sejam executados (AP 1.1) e gerenciados (AP 2.1). Depois (nível F), também, que os produtos de trabalhos sejam gerenciados. A partir do nível E, processos padrões devem ser definidos (AP 3.1) 
e implementados (AP 3.2). Nos níveis B e A os atributos de processo procuram garantir que os processos sejam controlados estatisticamente e otimizados continuamente.

Tabela 2 - Resultados Esperados Relacionados ao Processo Medição [SOFTEX 2009a]

\begin{tabular}{|l|l|}
\hline \multicolumn{1}{|c|}{ Sigla } & \multicolumn{1}{c|}{ Texto dos Resultados Esperados } \\
\hline GPR 2 & $\begin{array}{l}\text { As tarefas e os produtos de trabalho do projeto são dimensionados utilizando métodos } \\
\text { apropriados. }\end{array}$ \\
\hline GPR 4 & $\begin{array}{l}\text { (A partir do nível E) O planejamento e as estimativas das atividades do projeto são feitos } \\
\text { baseados no repositório de estimativas e no conjunto de ativos de processo organizacional. }\end{array}$ \\
\hline GPR 19 & $\begin{array}{l}\text { (A partir do nível E) Produtos de trabalho, medidas e experiências documentadas contribuem } \\
\text { para os ativos de processo organizacional. }\end{array}$ \\
\hline DFP 6 & O repositório de medidas da organização é estabelecido e mantido. \\
\hline AMP 10 & $\begin{array}{l}\text { Experiências relacionadas aos processos são incorporadas aos ativos de processo } \\
\text { organizacional. }\end{array}$ \\
\hline
\end{tabular}

A Tabela 3 apresenta alguns resultados esperados de atributo de processo relacionados ao processo Medição.

Tabela 3 -Resultados de Atributo de Processo Associados a Medição [SOFTEX 2009a]

\begin{tabular}{|c|c|l|}
\hline Sigla & AP & \multicolumn{1}{c|}{ Texto dos Resultados Esperados } \\
\hline RAP 4 & AP 2.1 & $\begin{array}{l}\text { (A partir do nível F) Medidas são planejadas e coletadas para monitoração da } \\
\text { execução do processo e ajustes são realizados. }\end{array}$ \\
\hline RAP 9 & AP 2.1 & $\begin{array}{l}\text { (Até o nível F) Os resultados do processo são revistos com a gerência de alto nível } \\
\text { para fornecer visibilidade sobre a sua situação na organização. }\end{array}$ \\
\hline RAP 21 & AP 3.2 & $\begin{array}{l}\text { Dados apropriados são coletados e analisados, constituindo uma base para o } \\
\text { entendimento do comportamento do processo, para demonstrar a adequação e a } \\
\text { eficácia do processo, e avaliar onde pode ser feita a melhoria contínua do processo. }\end{array}$ \\
\hline
\end{tabular}

\section{Observações e Melhores Práticas - Definição do Plano de Medição}

A implantação de um Programa de Medição não é trivial e, em geral, pode ser trabalhosa. Bons programas de medição começam a partir da derivação dos objetivos estratégicos da empresa para a definição de objetivos de medição (MED 1). De forma geral, esta associação possibilita que o esforço seja concentrado em áreas que contenham aspectos importantes para a tomada de decisão na organização como um todo e na área responsável pelo desenvolvimento de software em particular. Apesar de o foco do MPS.BR estar, de uma forma ampla, no desenvolvimento de software, isso não significa que a implementação do processo Medição não precise se preocupar em atender aos demais objetivos estratégicos da organização.

Assim, um dos principais problemas associados ao resultado esperado MED 1 é a falta de um plano estratégico na organização a partir do qual se possa derivar objetivos de medição. Muitas vezes, por causa de uma definição falha e ambígua deste plano, o relacionamento entre os objetivos estratégicos da empresa e os objetivos de medição é superficial e insuficiente para explicar completamente a associação entre eles. Desta forma, não se tem a garantia de que as medidas identificadas atendam corretamente às necessidades de informação dos diferentes níveis de gerência da empresa.

Algumas organizações ainda confundem Medição (como previsto pelo MRMPS) com o cálculo associado ao uso da técnica de Análise de Pontos por Função (APF) para determinar o tamanho de um produto (principalmente aquelas organizações em estágios iniciais do programa de melhoria). O cálculo do tamanho de um produto é apenas uma das medidas que podem ser utilizadas em uma organização e está associado principalmente à gerência de projetos (GPR 2) para definição de estimativas (GPR 4). Em geral, o tamanho de um produto é associado a outras medidas para formar indicado- 
res como a produtividade média de uma equipe ou densidade de defeitos.

O GQM (Goal-Question-Metric) [BASILI et al., 1994; SOLINGEN e BERGHOUT, 1999] é um método bastante utilizado para a definição de um Plano de Medição devido à sua simplicidade de uso alinhado com uma estrutura "topdown" que possibilita a identificação das medidas (M) a partir de questões (Q) associadas aos objetivos de medição $(\mathrm{O})$. Nem sempre, no entanto, o método é utilizado adequadamente, produzindo um conjunto de medidas que não são diretamente relacionáveis às questões e objetivos de medição, fato que prejudicará mais tarde a análise e a tomada de decisão.

Uma questão às vezes formulada é "quantas medidas são necessárias" para a implantação de um programa de medição (MED 2). Esta pergunta pode ser considerada "errada", pois o que importa, na verdade, é "quais medidas" podem auxiliar a empresa na tomada de decisão ou, pelo menos, em curto prazo, no conhecimento do comportamento de seus processos de software. Em geral, a maior parte das medidas a serem utilizadas pode ser obtida pela combinação de cinco medidas básicas [PUTNAM E MYERS, 2003]: tamanho (quantidade de funcionalidade, por exemplo, linhas de código, pontos de função), produtividade (quantidade de funcionalidade produzida em função do tempo e esforço despendido), tempo (duração do projeto), esforço (quantidade de trabalho despendido, ou seja, recurso/tempo) e confiabilidade (quantidade de defeitos).

Muitas vezes, a preocupação com o número de medidas é decorrente da necessidade de os processos serem monitorados a partir da coleta e análise de medidas (RAP 4, a partir do nível F). Uma organização pode definir um programa de medição com poucas medidas e elas serem efetivas para a monitoração dos processos. Por outro lado, é relativamente comum a definição de muitas medidas que, na prática, apenas dão muito trabalho para coleta e análise, mas produzem poucos resultados práticos.

Por exemplo, muitos questionam o custo/esforço de se ter medidas específicas para monitoração de cada um dos 17 processos do Nível C do MR-MPS (de forma a atender ao RAP 4). Uma solução às vezes adotada é associar a monitoração de alguns processos à execução de alguns processos de apoio, como, por exemplo, Garantia da Qualidade. Uma medida como "número de não conformidades em avaliações de qualidade do processo X" pode ser simples de coletar, mas não necessariamente apresenta dados suficientes para avaliar se o processo $\mathrm{X}$ está sendo efetivamente seguido ou útil para o projeto/empresa. Em alguns casos, pode indicar apenas se a implementação do processo Garantia de Qualidade está proporcionando que se encontre ou não defeitos nos documentos avaliados. Um problema ainda maior, neste caso específico, acontece quando os critérios de garantia da qualidade não asseguram de fato que aspectos relevantes dos documentos produzidos sejam avaliados. Por exemplo, para monitorar os processos Verificação (VER) e Validação (VAL) ter laudos de qualidade inadequados avaliando formato de títulos ou preenchimento de cabeçalhos, sendo, assim, incapazes de identificar problemas relevantes. Para surtir o efeito desejado, os critérios dos laudos de qualidade deveriam estar mais próximos de critérios de verificação (ou seja, observando o conteúdo dos documentos) do que critérios de garantia da qualidade simples (ou seja, observando a forma do documento). Além disso, se uma medida é utilizada para monitorar mais de um processo, deve-se uma visão independente de elementos de cada processo. No caso do exemplo anterior, por exemplo, seria mais adequado analisar o número e tipos de defeitos nas inspeções (VER) ou nos testes de aceitação (VAL).

De qualquer forma, a associação das medidas de monitoração dos processos também deve estar alinhada aos objetivos de negócio da organização, o que pode ser 
feito a partir, por exemplo, de um objetivo de medição de monitoração da qualidade dos processos de software (o que, afinal, é, ou deveria ser, um dos focos de programas de melhoria de processos baseados na adoção de modelos de maturidade). O esforço associado à implantação de um programa de medição é grande, assim, é importante que não sejam definidas muitas medidas para não se correr o risco de poucas serem realmente efetivas e não medirem nada relevante ou que, pelo seu alto custo de coleta e análise, sejam abandonadas ou ignoradas. Por outro lado, a definição de poucas medidas pode fazer com que aspectos realmente relevantes da execução dos processos sejam negligenciados e que as medidas sejam simples demais para a tomada de decisão. Deve-se cuidar para soluções simples não se transformem em soluções simplórias.

Uma vez identificadas as medidas necessárias para responder os objetivos de medição, cada uma delas deve ser totalmente definidas, incluindo os procedimentos de coleta e armazenamento (MED 3) e de análise (MED 4).

Os procedimentos de coleta devem ser definidos de tal forma que qualquer pessoa possa executá-los. Nem sempre é utilizado um formalismo adequado para isto. Uma boa forma de estruturação é encontrada na descrição operacional das medidas no PSM [McGARRY et al., 2001] ou na ISO/IEC 15939 [ISO/IEC, 2007]. É comum, por exemplo, a indicação que o valor de uma medida pode ser encontrado em uma ferramenta (por exemplo, o MS-Project), mas não informa-se como identificar de forma inequívoca a informação (por exemplo, que coluna do cronograma criado) ou quando a medida já pode ser coletada (por exemplo, só coletar o tempo esforço real da atividade se ela está $100 \%$ concluída). Outro problema comum é não descrever as medidas básicas (aquelas utilizadas para compor outras) isoladamente nem informar matemática e claramente as fórmulas de cálculo a serem utilizadas para derivar as medidas compostas.

Os procedimentos de armazenamento devem indicar o local exato onde as informações devem ser registradas. Isso inclui não somente onde os valores coletados serão transcritos (por exemplo, em que célula da planilha de coleta a quantidade de defeitos registrados em uma rodada de testes deve ser preenchida), mas também onde o instrumento de coleta (por exemplo, uma planilha de coleta) deve ser salvo.

Em relação aos procedimentos de análise das medidas é importante que eles realmente auxiliem a análise das informações. Por exemplo, informando em que momentos e por quem as medidas são analisadas e a frequência desta análise. Outro item relevante é indicar como combinar diferentes medidas para compreender o comportamento dos processos (por exemplo, relacionar o esforço despendido nas avaliações de qualidade com o número de não conformidades encontradas e o índice de retrabalho dos projetos). Uma omissão comum dos procedimentos de análise é a falha em indicar a necessidade de se separar: dado da medida, informação de contexto, a análise de fato e as ações decorrentes das análises realizadas.

A falta de metas (apesar de não obrigatórias) para as medidas é, também, uma característica que pode dificultar análises. Mesmo em organizações iniciando o programa de medição (e que, portanto, não têm uma base histórica) podem se beneficiar da definição de metas. Com o tempo, se as metas forem consideradas inadequadas, podem ser revistas. Mas a existência delas possibilita alguma visão crítica dos valores obtidos.

Quando uma organização está iniciando o seu programa de melhoria é comum que não haja uma base histórica, portanto, a comparação de valores passados pode não ser eficiente. Por outro lado, em empresas com maior maturidade, a comparação de va- 
lores passados é um importante mecanismo para a tomada de decisões, por exemplo, a partir da análise de tendências do número de defeitos nos testes ou evolução da produtividade nos projetos. No entanto, análises mais elaboradas muitas vezes são negligenciadas pelas organizações. Isto é crítico, principalmente, em organizações a partir do Nível E, onde a existência de processos padrão possibilita tais comparações.

A partir do Nível E, um novo requisito do modelo é a existência de um repositório de medidas na organização (DFP 6). Um repositório de medidas deveria ser capaz de registrar todas as medidas (de acordo com o plano de medição elaborado) coletadas na organização e possibilitar o seu uso futuro. Considerando a adoção do MR-MPS de forma evolutiva em uma organização, isso é importante principalmente para os níveis de alta maturidade (A e B) que requerem tratamento diferenciado para os dados de medição e o controle estatístico dos processos. Para o efetivo controle estatístico dos processos, os dados analisados não podem conter erros e devem ser armazenados de forma a conservar informações de contexto, relacionamentos entre medidas e serem manipuláveis (por exemplo, com a derivação de outras medidas compostas) adequadamente. Isto não será conseguido facilmente em organizações cujos repositórios de medidas sejam armazenados em planilhas Excel ou em apresentações do PowerPoint (o que é mais comum do que se imagina).

Um Plano de Medição é um importante ativo da organização e, como tal, deve ser gerenciado adequadamente, o que inclui controle de acesso e mecanismos de gerência de configuração. Nem sempre isso é adequadamente implementado pelas organizações, algumas vezes se encontram organizações que definem suas medidas apenas em atas de reunião não formalizando em um documento específico.

\section{Observações e Melhores Práticas - Execução da Medição}

O Plano de Medição elaborado deve ser posto em prática através da coleta e análise das medidas (MED 5) planejadas de acordo com a frequência definida. Todas as medidas devem ser coletadas e analisadas de acordo com o planejado (se os procedimentos definidos não forem adequados, devem ser revistos, não ignorados). Algumas análises podem ser mais frequentes que outras e isto deve ser definido caso a caso. Por exemplo, na maior parte das vezes não justifica analisar o percentual de atraso de um projeto apenas após ele ter sido concluído, por outro lado, analisar a satisfação dos clientes quinzenalmente pode não ser razoável.

É importante que se utilizem gráficos adequados para a visualização dos valores das medidas. Um problema comum é o uso de histograma para dados que não são séries históricas (por exemplo, para comparação do número de defeitos de cada projeto em execução em um mês é melhor utilizar um gráfico de barra do que um histograma). Os procedimentos de análise deveriam informar quais gráficos devem ser gerados e como devem ser interpretados. Deve-se procurar evitar análises pouco profundas, que apenas repitam o que o gráfico diz sem acrescentar nada além.

Outros cuidados que deveriam ser adotados incluem a ordenação adequada dos valores (dados históricos, por exemplo, deveriam estar em ordem de data e não em ordem alfabética) e implementação de procedimentos de garantia de qualidade para assegurar que os dados coletados estão corretos e representam os valores associados às propriedades das entidades medidas. Da mesma forma, é importante que as análises realizadas sejam auditadas pela Garantia da Qualidade para assegurar que o Plano de Medição está sendo seguido. É comum em algumas organizações que as análises fiquem re- 
gistradas apenas em atas de reunião e que não sejam avaliadas pela Área de Qualidade. Desta forma, negligencia-se um importante aspecto do processo Medição e prejudica-se, também, a adequada institucionalização do processo Garantia da Qualidade.

O armazenamento das análises (MED 6) é importante como fonte histórica da evolução do programa de medição e também para registrar as decisões tomadas. Como todo documento importante, os registros de medição deveriam ser armazenados sob algum nível de controle de gerência de configuração. Um item importante das análises das medidas são as informações de contexto, que devem possuir o máximo de detalhes para possibilitar comparações mais efetivas no futuro e, também, identificar medidas com viés. Por exemplo, se as informações de contexto forem perdidas, com o tempo a análise de uma longa série histórica das medidas será comprometida porque dificilmente as pessoas se lembrarão de fatos ocorridos há muito tempo.

Se uma medida é coletada ela, em geral, ou deve ser utilizada para compor outra medida ou, então, ser analisada e divulgada (MED 7) por meio eletrônico ou presencial. Quanto mais frequentemente as medidas forem analisadas, mais rápida e efetiva pode ser a tomada de decisão. A falha em tomar decisões rápidas pode fazer com que o esforço do programa de medição seja em vão e prejudicar seu apoio pelos envolvidos. Além disso, reuniões de discussão de medidas com periodicidade grande (e. g., superior a 4 meses) podem ser improdutivas, principalmente em organizações em estágios iniciais do programa de melhoria de processos. Deve-se evitar também a discussão de todas as medidas com todos os envolvidos indistintamente. Deve-se procurar discutir apenas medidas pertinentes com cada perfil de profissional senão corre-se o risco de desinteresse. Por exemplo, diferenciar medidas de interesse da alta gerência (e.g., lucratividade média dos projetos) daquelas mais próximas dos profissionais envolvidos na execução dos projetos (e.g., número de defeitos encontrados nos testes de integração).

Como as medidas são originadas dos objetivos de medição, as análises também deveriam relacionar tais objetivos com os valores obtidos. $\mathrm{O}$ apoio da alta gerência pode ser enfraquecido em caso de falha na identificação deste relacionamento, o que pode colocar em risco todo o programa de medição causando até o seu abandono. As reuniões de discussão dos resultados das medições também podem ser bons momentos para apresentar à alta gerência resultados da iniciativa de melhoria de processos e avaliar a institucionalização dos processos (RAP 9). Os produtos gerados em decorrência da execução do processo Medição também permitem constituir uma base para o entendimento do comportamento dos processos (RAP 21) e podem auxiliar a direcionar melhor os esforços do programa de melhoria de processos da organização (AMP 10).

De pouco adianta a coleta e análise das medidas se não há tomada de decisão (MED 7) associada a elas. As ações decorrentes das medidas devem ser gerenciadas até a conclusão; a falha em cumprir tais determinações também pode ser um indicativo da perda de prioridade e importância do programa de medição.

\section{Observações e Melhores Práticas - Base Histórica de Estimativas}

O processo Medição, como um processo de apoio, também pode auxiliar a implementação de outros processos na organização, como, por exemplo, contribuindo para a coleta de informações e medidas (GPR 19) necessárias definição de uma base de estimativas e seu calibramento para a realidade da organização.

Neste caso, também, são necessários alguns cuidados pra evitar distorções e tomada de ações com base em dados incorretos. Dentre eles: (i) utilizar medidas coletadas 
adequadamente a partir dos dados dos projetos (por exemplo, duração das atividades e tamanho do projeto); (ii) evitar consolidar dados de projetos muito diferentes (por exemplo, pequenos e grandes, de diferentes linguagens, utilizando versões diferentes dos processos padrão ou, então, projetos muito antigos com novos); (iii) calcular corretamente o tamanho do produto (por exemplo, seguir o formalismo associado a uma contagem de Pontos por Função); (iv) calcular o tamanho real do produto ao final do projeto e não utilizar o valor estimado no início do projeto; (v) ter claramente definidas as atividades do processo de desenvolvimento da empresa (evitando que o cronograma dos projetos não seja fielmente baseado no processo padrão da organização); e (vi) garantir que as medidas presentes na base de dados sejam confiáveis (por exemplo, através da adoção de mecanismos de garantia da qualidade para avaliá-las).

\section{Considerações Finais}

Este artigo apresentou um conjunto de observações relacionadas ao estado da prática da implantação de um Programa de Medição em organizações de software. Apesar de as observações aqui listadas, em geral, apresentarem problemas recorrentes, há bons exemplos de implementação de programas de Medição em diferentes organizações.

As observações presentes neste artigo não pretendem ser substitutas a outras fontes de informação sobre Medição de Software ou aos Guias de Implementação do MR-MPS [SOFTEX 2009b]. Espera-se, no entanto, que organizações possam utilizá-las na estruturação e condução de suas iniciativas de melhoria de processos de software de forma a otimizar os esforços e evitar erros e falhas comumente cometidos.

\section{Agradecimentos}

$\mathrm{O}$ autor agradece à FAPERJ pelo auxílio financeiro.

\section{Referências Bibliográficas}

BASILI. V.; CALDIERA, G.; ROMBACH, H., 1994, "Goal Question Metric Paradigm”, In: Encyclopedia of Software Engineering, v.2, pp: $527-532$.

ISO/IEC - International Organization for Standardization and the International Electrotechnical Commission, 2007. ISO/IEC 15939: System engineering - Software measurement process framework, Geneve: ISO.

McGARRY, J., CARD, D., JONES, C. et al., 2001, Practical Software Measurement: Objective Information for Decision Makers, Addison-Wesley.

PUTNAM, L. H. e MYERS, W., 2003, Five Core Metrics: The Intelligence Behind Successful Software Management, Dorset House Publishing Company.

SANTOS, G. et al. (2009a), "Lições Aprendidas em uma Iniciativa de Melhoria de Processos de Software sob Diferentes Perspectivas: Membros da Organização, Implementadores e Avaliadores". In: VIII Simpósio Brasileiro de Qualidade de Software - SBQS 2009, Ouro Preto - MG, Junho 2009.

SANTOS, G. et al. (2009b), "Indicadores da Implementação do Nível E do MR-MPS em uma Instituição de Pesquisa". In: VIII Simpósio Brasileiro de Qualidade de Software - SBQS 2009, Ouro Preto - MG, Junho 2009.

SOFTEX (2009a) "MPS.BR: Melhoria de Processo do Software Brasileiro - Guia Geral", Disponível em: http://www.softex.br/mpsbr, último acesso em 18/04/2011.

SOFTEX (2009b) "Guia de Implementação - Parte 2: Fundamentação para Implementação do Nível $F$ do MR-MPS", Disponível em: http://www.softex.br/mpsbr, último acesso em $18 / 04 / 2011$.

SOLINGEN, R.; BERGHOUT, E., 1999, The Goal/Question/Metric Method: a Practical Guide for Quality Improvement of Software Development, McGraw-Hill. 